\title{
Recovery of the 6-year signal in length of day and its long-term decreasing trend
}

\author{
Pengshuo Duan ${ }^{1,2^{*}}$, Genyou Liu', Lintao Liu', Xiaogang Hu', Xiaoguang Hao ${ }^{1}$, Yong Huang ${ }^{1,2,3}$,
} Zhimin Zhang ${ }^{1,2}$ and Binbin Wang ${ }^{1,2}$

\begin{abstract}
There is a significant 6-year oscillation signal (called 6-year signal in this paper) existing in the interannual variations of length of day (LOD). It is unclear to understand its nature variation features. This paper extracts quantitatively the 6-year signal, from 1962 2012, using normal Morlet wavelet (NMWT) method combining wavelet packet and Fourier analysis technique, for the first time, and we investigate it in both time and frequency domains. The results indicate that the amplitude of a 6 -year signal shows a long-term decreasing trend and the total amplitude reduction is about $0.05 \mathrm{~ms}$ during the past 50 years. The ratio of above reduction to the mean amplitude of $0.124 \mathrm{~ms}$ reaches $40 \%$. For interpreting the phenomenon on the above long-term decreasing trend, this paper proposes two alternatives; however, there is still no firm conclusion and it is required to be further explored.
\end{abstract}

Keywords: Length of day; Long-term decreasing trend; NMWT method; Background trend

\section{Background}

Variations of length of day (LOD) have a wide spectral range of periods, which contains seasonal signals (e.g., annual and semiannual), interannual, and decadal variations (Chao et al. 2014; Zheng et al. 2000). The seasonal variations are excited by atmospheric, ocean, and land hydrology (Chen 2005); the above decadal term is supposed to be caused by core-mantle coupling and interaction (Mandea et al. 2010; Mound and Buffett 2003; Wang et al. 2000). However, the interannual variations are less clear (Holme and de Viron 2013). Many publications indicate that a large part of the interannual variations can be attributed to the atmospheric angular momentum (AAM) (Chen 2005; Chao and Yan 2010) or the combined effects of EI Niño-Southern Oscillation (ENSO) and quasi-biennial oscillation (QBO) (Chao 1989). However, the geophysical mechanism of the signals in lower frequency band on LOD interannual scales (e.g., the obvious 6-year signal) is still unknown. Although gravitational coupling is most likely (Mound and Buffett 2006), numerous previous studies speculated the

\footnotetext{
* Correspondence: duanpengshuo12@163.com

${ }^{1}$ Institute of Geodesy and Geophysics, Chinese Academy of Sciences, State Key Laboratory of Geodesy and Earth's Dynamics, Wuhan 430077, China

${ }^{2}$ University of Chinese Academy of Sciences, Beijing 100049, China

Full list of author information is available at the end of the article
}

above geophysical mechanism may stem from the Earth's internal core-mantle electromagnetic coupling (Abarco del Rio et al. 2000, 2003; Holme and de Viron 2005, 2013; Gorshkov 2010; Silva et al. 2012; Davies et al. 2014). Consequently, the topic on geophysical mechanism of the 6-year signal has been controversial up to date. On the other hand, the interpretations of the nature variation feature of the 6-year signal are nonunique (Abarco del Rio et al. 2000, 2003; Holme and de Viron 2013; Gorshkov 2010). For instance, Abarco del Rio et al. (2000) made the first robust observation of power near the 6-year period signal with a mean amplitude of $0.12 \mathrm{~ms}$ in the LOD series based on wavelet analysis and singular spectrum analysis (SSA); actually, the result from Abarco del Rio et al. (2000) has indicated that the signals on 6 7-year scales show a decreasing phenomenon, but which was not described; Gorshkov (2010) indicated that the 6 7-year oscillation signals decreased abruptly in the 1990s and speculates it is due to the stronger signals in a 2 3-year band canceling out the 6 7-year signals; while, Holme and de Viron (2013) further indicated that the "6-year oscillation" shows an apparent drop in amplitude in the 1990s (but this amplitude returns to full strength in the next cycle), and they interpreted it as the consequence of the LOD decadal background trend in the 1990s.

\section{勿 Springer}


To make the geophysical mechanism of the 6-year signal be clear, firstly, we have to extract accurately the 6-year signal and study its own nature variation features precisely (including its instantaneous amplitude, phase, and periodic), in both time and frequency domains. For resolving the above problem, this paper studies quantitatively the monthly LOD data from 1962 to 2012, using normal Morlet wavelet (NMWT) method (Liu et al. 2007) combining orthogonal Daubechies wavelet (Daubechies 1988) with higher-order vanishing moment based on wavelet packet analysis and Fourier analysis technique. NMWT is very suitable for harmonic analysis even if the instantaneous amplitude of the harmonic signal to be analyzed is slowly varying with time (Liu et al. 2007). We extract the 64-96-month (5.3-8-year) band signals based on wavelet packet analysis from original LOD series, which shows an obvious decreasing phenomenon in the 1990s. The above phenomenon is identical to the published results (Abarco del Rio et al. 2000; Holme and de Viron 2013; Gorshkov 2010). But, the signal extracted by wavelet packet analysis contains more frequency components due to it covering a wide frequency range so that it cannot be a perfect representative of the actual 6-year signal. Fortunately, this paper indicates that we can obtain the signal with a quite narrow frequency range and accurate phase using NMWT method and its energy concentrates at 0.0138 cycles per month (cpm). In this work, we will show how to recover the target 6-year signal using NMWT method in detail.

\section{Methods}

\section{Data and resources}

The LOD data this paper uses is loaded from the website: http://www.iers.org/IERS/EN/ Data/Earth Orientation Data; the EOP 08 C04 series time span is from 1962 to 2012 and the sampling interval is 1 day. We get the monthly LOD data by using the monthly averaging method. AAM monthly data from 1962 to 2012 can be loaded from the website: http://www.cpc.ncep. noaa.gov. Here, the AAM data is the total angular momentum, which contains two terms: motion term (wind excitation) and mass term (pressure excitation). The relation between $\triangle \mathrm{AAM}$ and $\Delta$ LOD is as follows (Chao and Yan 2010)

$$
\Delta L O D=\frac{86400}{C_{m} \Omega} \times \triangle \mathrm{AAM}
$$

where $\Omega$ is the Earth mean rotation rate and $C_{m}$ is the principal moment of inertia of the Earth's mantle of about $7.1 \times 10^{37} \mathrm{kgm}^{2}$.

\section{NMWT method and its two properties}

The continuous wavelet transform (CWT) has been widely used in signal processing. For the time signal $f(t) \in L^{2}(R)$, the CWT form is as follows

$$
W_{f}(a, b)=\frac{1}{\sqrt{|a|}} \int_{R} f(t) \bar{\psi}\left(\frac{t-b}{a}\right) d t
$$

where $a$ and $b$ are scale and time translating indices, respectively; $\psi$ is the analysis wavelet or admissible wavelet kernel function; and the horizontal line signifies the function conjugate. The admissible condition of wavelet is

$$
c_{\psi}=2 \pi \int_{-\infty}^{+\infty} \frac{|\hat{\psi}(\omega)|^{2}}{|\omega|} d \omega<+\infty
$$

where the sign $\wedge$ is the Fourier transform operator. When the kernel function is Morlet wavelet, the continuous wavelet transform is called Morlet wavelet transform. The traditional Morlet wavelet (which meets the admissible condition) is given by

$$
\psi(t)=\left[\exp \left(-\frac{\tau^{2}}{2 \sigma^{2}}\right)-\sqrt{2} \exp -\left(\frac{\tau^{2}}{\sigma^{2}}+\pi^{2} \sigma^{2}\right)\right] e^{i 2 \pi \tau}
$$

where $\sigma$ is the window width operator of wavelet and the unit of $\sigma$ is month. Formula (4) tells us that the traditional Morlet wavelet is designed to be composed by two Gauss functions; the aim of doing this is only to make the analysis wavelet meet the wavelet admissible condition. However, NMWT alters the traditional Morlet wavelet basis function, and it is different from the traditional Morlet wavelet transform. The reformed Morlet wavelet is

$$
G(\tau)=\exp \left(-\frac{\tau^{2}}{2 \sigma^{2}}+i 2 \pi \tau\right)
$$

Here, $G(\tau)$ is more simple. If $\sigma$ is large enough, $G(\tau)$ will be close to admissible wavelet. Then, a mathematical transformation called normal Morlet wavelet transform (i.e., NMWT) based on the simplified $G(\tau)$ wavelet basis in $L^{1}(R)$ space has been defined. NMWT is especially suitable for harmonic and quasi-harmonic analysis, which is due to NMWT owning two extremely important properties and another two obvious following virtues as well, i.e., (1) NMWT can identify and extract the target signals from the original time series without inverse transform; (2) Phase shifting of the signals extracted by NMWT does not happen. Liu et al. (2007) studied the harmonic signals in the polar motion based on NMWT method, and they got a very ideal result. This paper will study and recover quantitatively the 6-year signal in LOD through further combining NMWT, wavelet packet analysis, and Fourier analysis technique. This paper uses 
the reformed Morlet wavelet $G(\tau)$ to construct the normal Morlet wavelet $g(\tau)$, the definition form is given by

$$
g(\tau)=G(\tau) / \hat{G}(2 \pi)=\frac{1}{\sqrt{2 \pi} \sigma} \exp \left(-\frac{\tau^{2}}{2 \sigma^{2}}+i 2 \pi \tau\right)
$$

where the sign $\wedge$ is the Fourier transform operator; $\sigma$ reflects the window width of normal Morlet wavelet and the larger $\sigma$ can guarantee the higher-frequency resolution. This paper takes $\sigma=3$. For $f(t) \in L^{1}(R)$, NMWT is defined as follows

$$
W_{g} f(a, b)=\frac{1}{|a|} \int_{-\infty}^{+\infty} f(t) \bar{g}\left(\frac{t-b}{a}\right) d t
$$

where $a, b \in R, a \neq 0$, and

$$
L^{1}(R)=\left\{f(t)|| \int_{-l}^{+l} f(t) d t \mid<+\infty, \forall l \in R^{+}\right\}
$$

Assuming that the mathematical form of the 6-year harmonic signal to be analyzed can be expressed as the final complex formula $f(t)=A \exp \left(i \omega\left(t-t_{0}\right)\right)$, where $i$ is the imaginary unit and $\omega=\frac{2 \pi}{T}$, then, the two important properties of NMWT are as follows (In this article, it is unnecessary to repeat the proof process, which is shown in detailed by Liu et al. 2007)

Property 1: $W_{g} f(T, b)=f(b),(\forall t=b, a=T)$; Property 2: $\frac{\partial}{\partial a}\left|W_{g} f(a, b)\right|=0,(\forall a=T)$;

Property 1 demonstrates that, for the periodic function $f(t)$, we apply NMWT to $f(t)$, then the wavelet coefficients (at $a=T$ ) equal to signal $f(b)$ with $\forall t=b$ exactly. That is to say both of the correct instantaneous phase and amplitude are precisely recovered, which is unrelated with $\sigma$.

Property 2 demonstrates that, when $b$ is fixed, $\mid W_{g} f(a$, $b) \mid$ spectrum can get local maxima at $a=T ;\left|W_{g} f(a, b)\right|$ spectrum along the locations of local maxima, with $b$ changing, generates a line called harmonic "ridgeline"; following the "ridgeline", we can recognize the harmonic signal with periodic $T$. Extracting the NMWT values that the "ridgeline" corresponds to, we can recover the harmonic signal, which is also unrelated with $\sigma$.

The above two properties of NMWT are the theoretical basis of extracting precisely the harmonic signal from original time series. Hence, they are very useful and important. Furthermore, NMWT method is also applicable for analyzing the quasi-harmonic signals with a slowly and slightly varying amplitude and frequency (Liu et al. 2007). Consequently, NMWT method should be very suitable for studying accurately the 6-year signal in LOD series.

\section{Results}

Wavelet transform owns the edge effect (Zheng et al. 2000), which can bring some errors into the final results. In order to study the 6-year signal using NMWT method quantitatively, we need to eliminate the edge effect. The commonly effective method of eliminating the edge effect is to make a symmetrical extension at the two boundaries of LOD data (see Fig. 1). We can get the interannual variations (see the blue curve in Fig. 1) after filtering the seasonal signals from the residual series, where the residual series referring to the decade has been removed from the original series. The red curve is the decade term; the green curve is the residual of decade subtracted from original LOD; and the blue curve is interannual variations that seasonal and decade terms

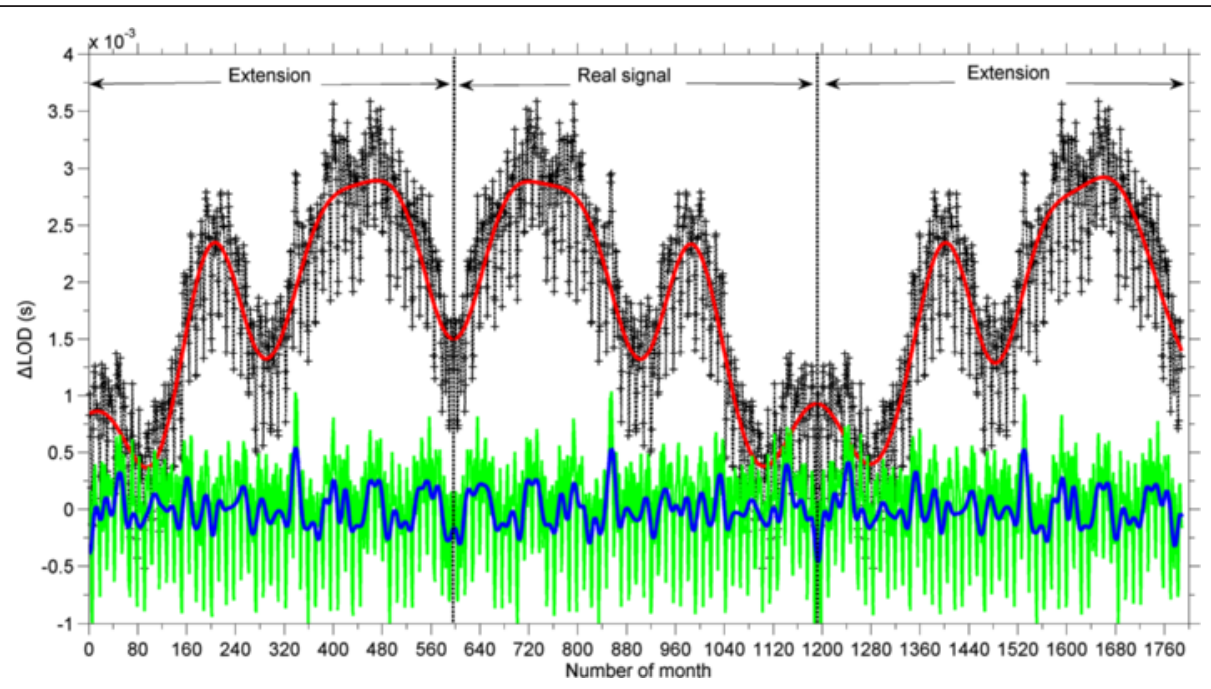

Fig. 1 The original LOD series and its various processing results after symmetric extension at the two boundaries 


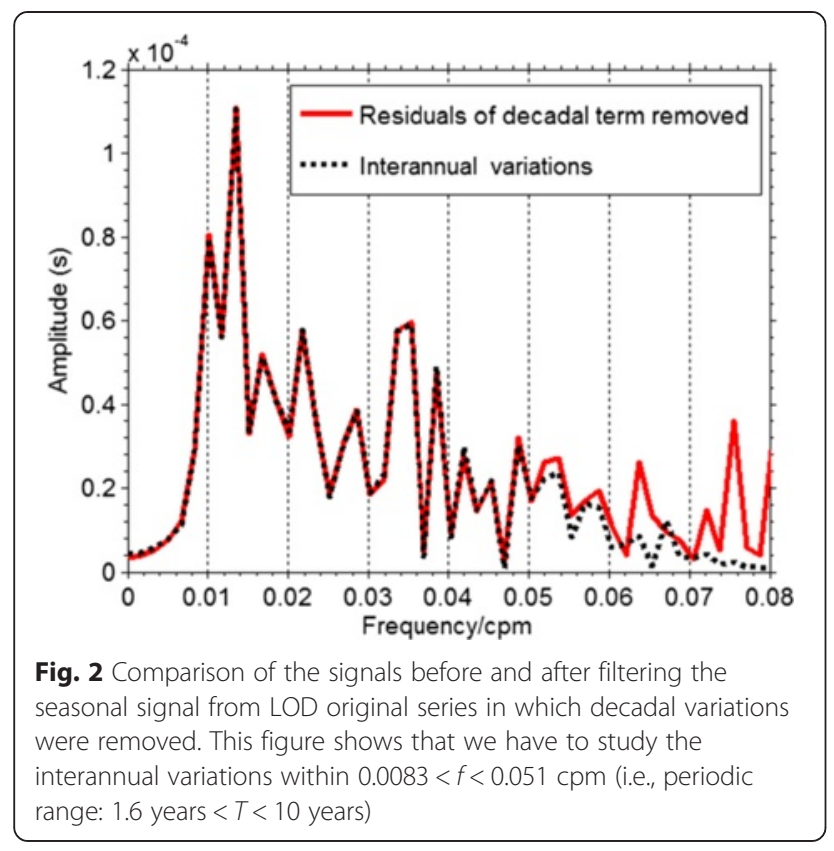

have been removed. "Real signal" means the original LOD series from 1962 to 2012; "Extension" refers to the symmetric extension parts. But we have to test whether the target interannual signal is disturbed or not during the above filtering process. Consequently, we make a further detailed comparison of the signal before and after filtering (see Fig. 2). Figure 2 shows that the frequency spectrum of the above two signals is in exact agreement with each other within the frequency scope $0.0083 \mathrm{cpm}<f<0.051 \mathrm{cpm}$.
Numerous publications show that AAM (motion term + mass term) is a primary contributor to interannual variations in LOD (Abarco del Rio et al. 2000, 2003; Holme and de viron 2013; Chen 2005; Chao and Yan 2010). Consequently, we should remove the AAM effect from the original LOD series. Figure 3a shows that we can divide the spectrum of original LOD series into two frequency bands, i.e., $1.6-5$ years and 56.7 years. Comparing Fig. 3a with Fig. 3b, we can see that, after the AAM was removed, the signals in 1.6 5year band are greatly weakened while the 5 6.7-year signals are almost uninjured but the 6-year signal showing more notable.

To further investigate the harmonic feature of the 6year signal, we take the modulus of $W_{g} f(a, b)$ (NMWT spectrum), i.e., $\left|W_{g} f(a, b)\right|$ (see Fig. 4). Where $W_{g} f(a, b)$ means applying NMWT to time signal $f(t)$ (i.e., the LOD time series on the interannual scales); $g$ signifies the normal Morlet wavelet; and $a$ and $b$ are scale factor and translating index, respectively. From Fig. 4, we can see the continuous and stable "ridgeline" (the thin black lines are the local maxima, and they represent the instantaneous frequency or periodic variations) for the corresponding quasi-harmonics in $\left|W_{g} f(a, b)\right|$ spectrum on 1.6-6.7-year scales. Consequently, it is valid to see the 6-year signal as a quasi-harmonic signal according to the viewpoint from Liu et al. (2007) that NMWT values along the "ridgeline" recovering the corresponding quasi-harmonics. Figures 5 and 6 are respectively the corresponding 6-year quasi-harmonics signals from original series and the AAM removed series, which are

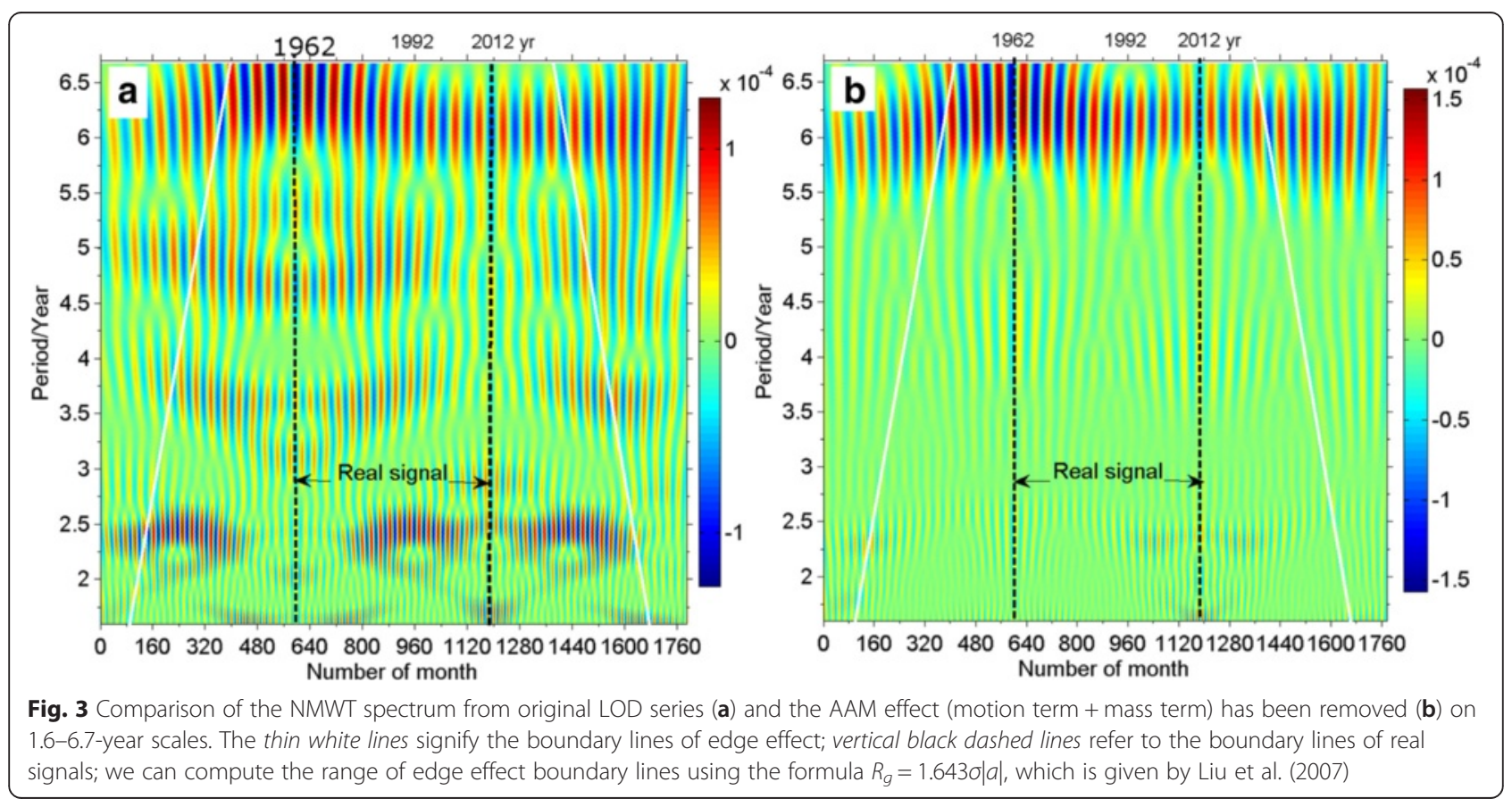




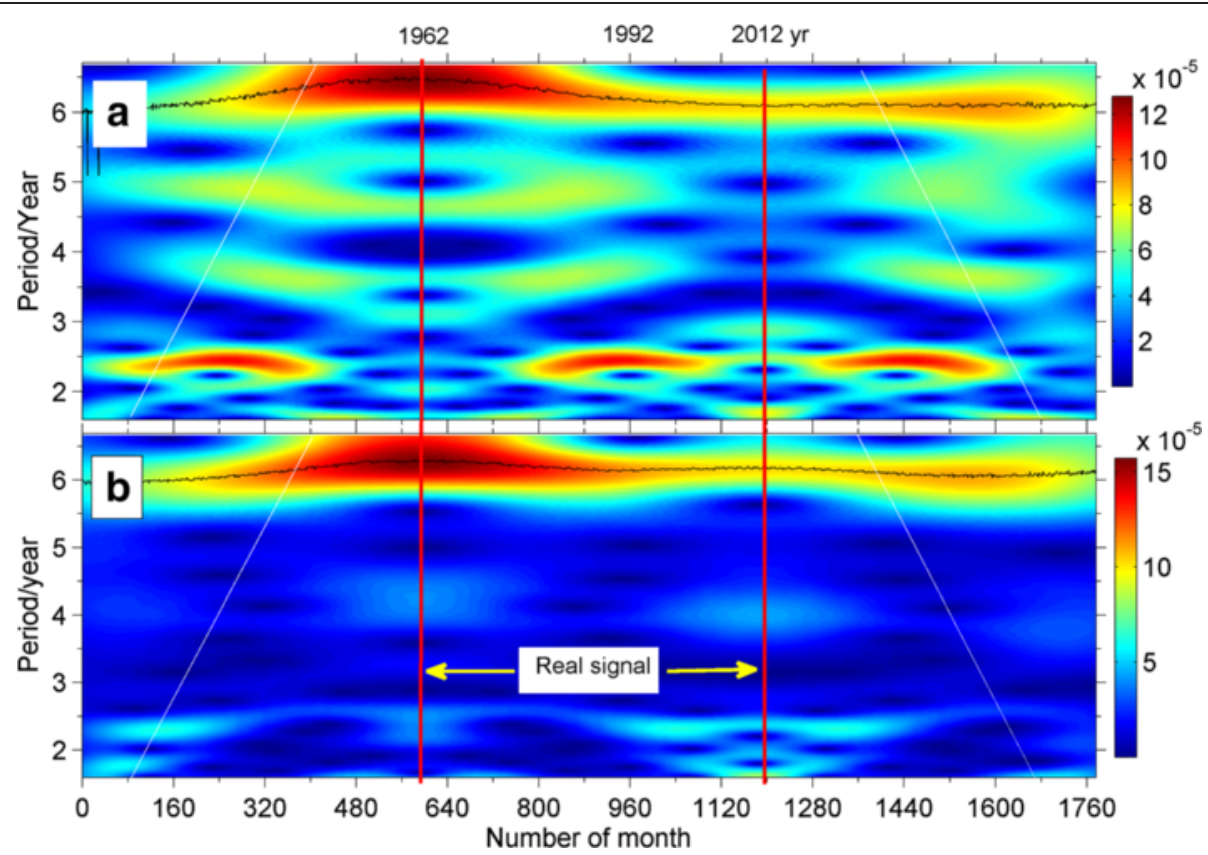

Fig. 4 Comparison of the $\left|W_{g} f(a, b)\right|$ spectrum from a original LOD series and $\mathbf{b}$ the AAM effect (motion term + mass term) has been removed, on 1.6-6.7-year scales. The black lines refer to "ridgeline", and the location of "ridgeline" shows the instantaneous periodic of the target signals. We can recover the target 6-year signal through extracting the wavelet transform coefficient real part values that the "ridgeline" corresponds to, from the NMWT spectrum, without inverse transformation, directly and quantitatively

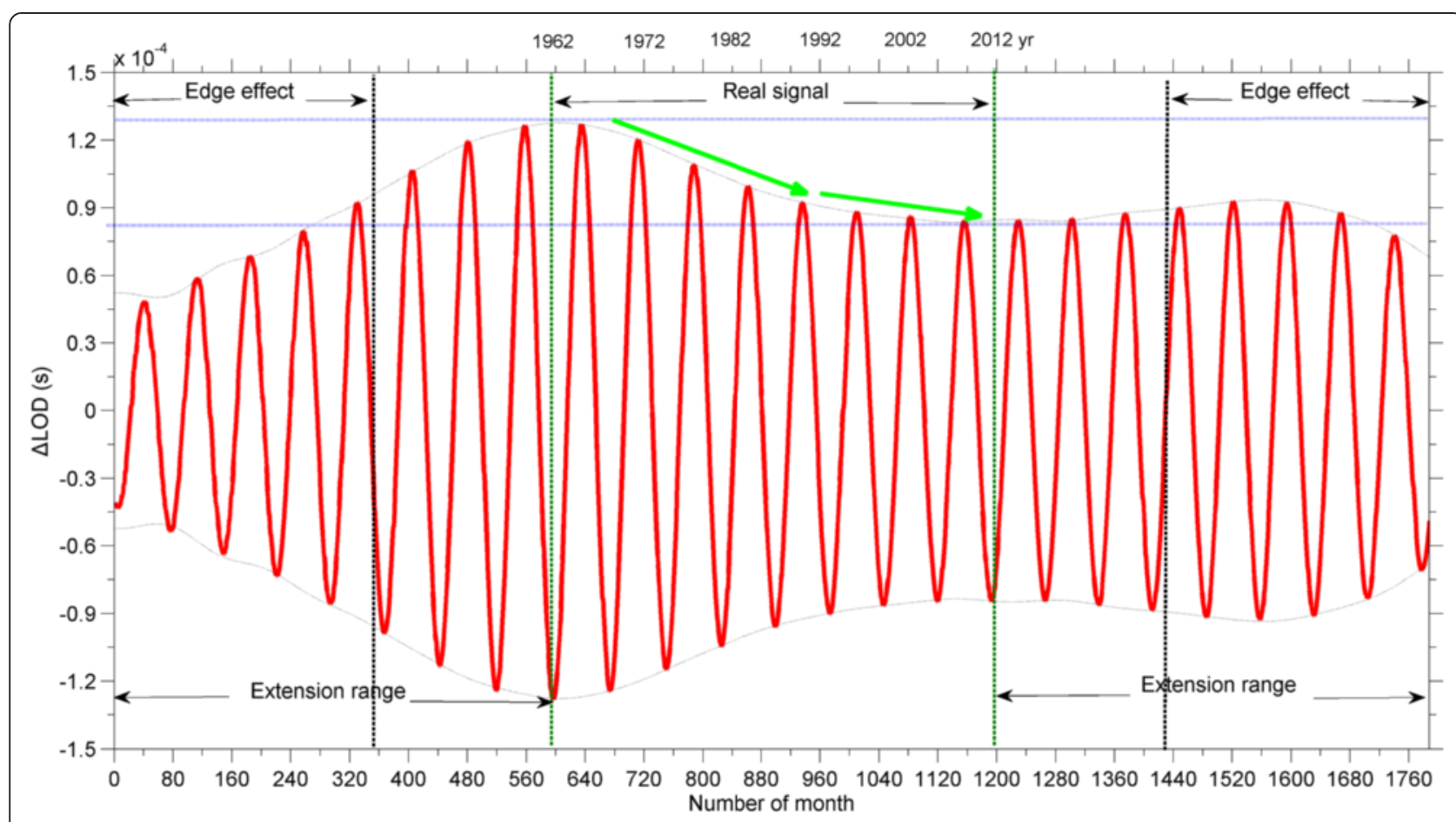

Fig. 5 The 6-year signal in original LOD series after a decade was removed and symmetric extension at the two boundaries. This figure indicates the 6-year signal (real signal) is showing a long-term decreasing trend 


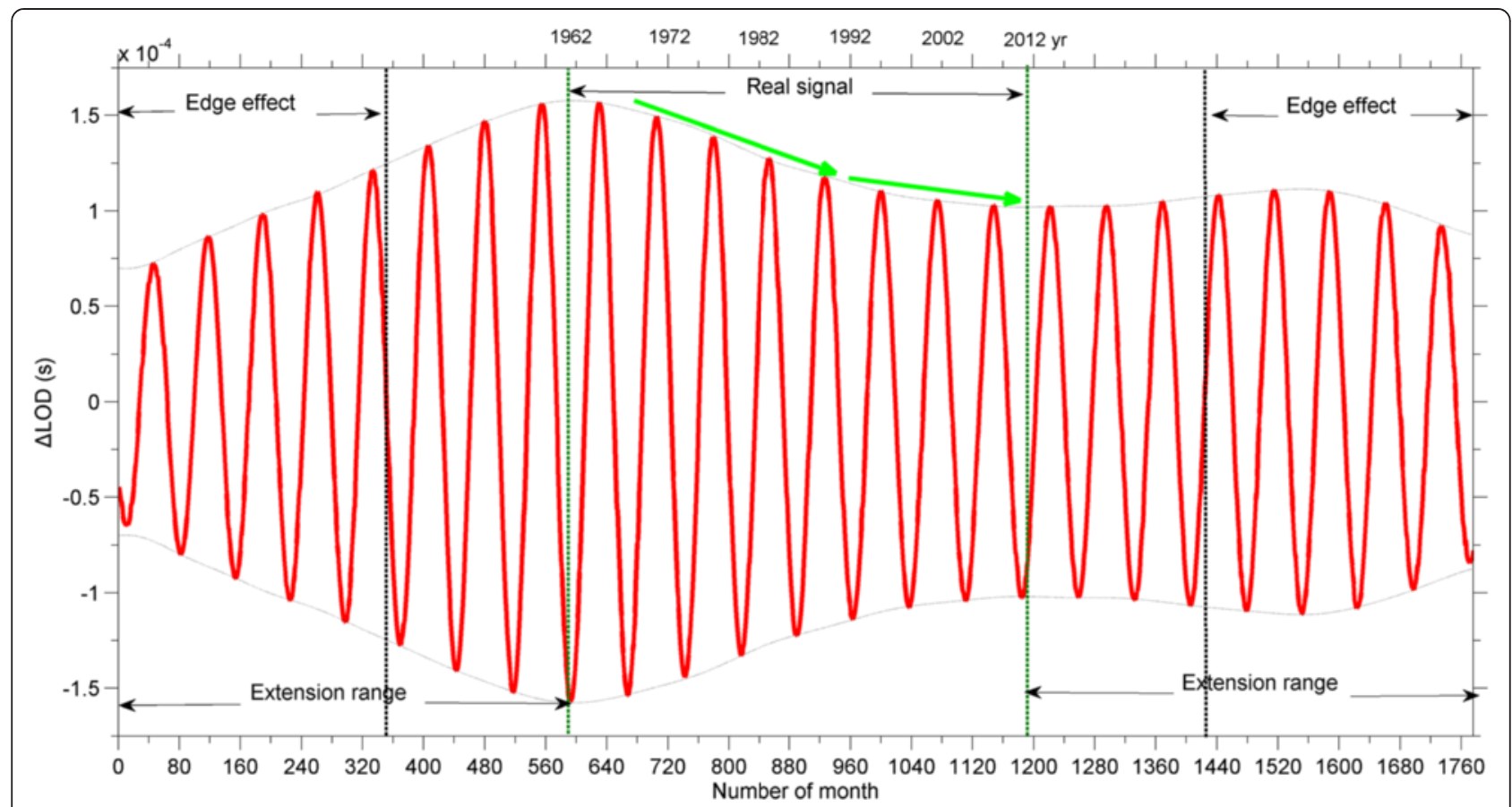

Fig. 6 The 6-year signal in LOD series (in which AAM effect has been removed) after a decade was removed and symmetric extension at the two boundaries

both extracted by NMWT. Obviously, the target 6-year signal (i.e., the real signals present in Figs. 5 and 6) shows the decreasing trend in the whole, which is unrelated to the wavelet edge effect.

In order to ensure the accuracy of the target signal extracted by NMWT and verify the superiority of NMWT method in extracting signals, we extract the target signal using two methods respectively and then make a detail comparison of the above two results in both time and frequency domains. The first method is using wavelet packet method based on the orthogonal Daubechies wavelet with higher-order vanishing moment; where the wavelet packet method can provide a fine analysis of the signal (the detailed principle of wavelet packet analysis will not be repeated again in this paper). According to the principle of wavelet packet analysis and the sampling rate of LOD data (30 days) in this paper, we can obtain the signals in a 64 96-month band (i.e., 5.3 8 years) (see Fig. 7a). Here, the order of (Daubechies wavelet) vanishing moment $(N)$ is 45 and the corresponding compactly supported length is 89 . Choosing the higher order of vanishing moment can help us extract signals with more accuracy (Daubechies 1988; $\mathrm{Hu}$ et al. 2005, 2006). The second method is using NMWT method; we extract the instantaneous values that the "ridgeline" corresponds to, from NMWT spectrum (Liu et al. 2007); thus, we can get the target signal with accurate phase in the time domain, whose frequency spectrum peak is very close to $0.0138 \mathrm{cpm}$ (see Fig. 7a, b).
Figure 7a indicates that the signal from wavelet packet method shows an obvious decreasing phenomenon in the 1990s, which is consistent with a previous study (Abarco del Rio et al. 2000; Holme and de viron 2013; Gorshkov 2010); however, Fig. 7a also shows the signal based on NMWT is gradually weakening during 19622012 instead of abrupt change in some period. Figure 7b reveals the three Fourier amplitude spectrum peaks (all values are about $0.104 \mathrm{~ms}$ ) locating at $0.0138 \mathrm{cpm}$, from original signal and wavelet packet and NMWT, are very consistent with each other; but, obviously, the signal with the most concentrated energy and the most narrow frequency range and no side-lobe interference and the very ideal filtering effect is from NMWT method.

Figure 8a shows the variation feature of the 6-year signal in which AAM effect has been removed. The long-term reduction is about $0.5 \times 10^{-4} \mathrm{~s}(0.05 \mathrm{~ms})$ during 1962 2012 years. The ratio of the above reduction $(0.05 \mathrm{~ms})$ to the mean amplitude of $(0.124 \mathrm{~ms})$ reaches about $40 \%$. Figure $8 \mathrm{~b}$ indicates that the magnitude of the 6-year signal with a mean amplitude of $0.124 \mathrm{~ms}$ concentrates closely at the $0.0138 \mathrm{cpm}$, which demonstrates the 6-year signal based on NMWT method should be reliable and correct. Talking about the above, the concept about long-term variation of the 6-year signal extracted by NMWT method can be defined as follows: the total envelope curve variation trend of the 6-year signal is called the long-term variation of this signal. 

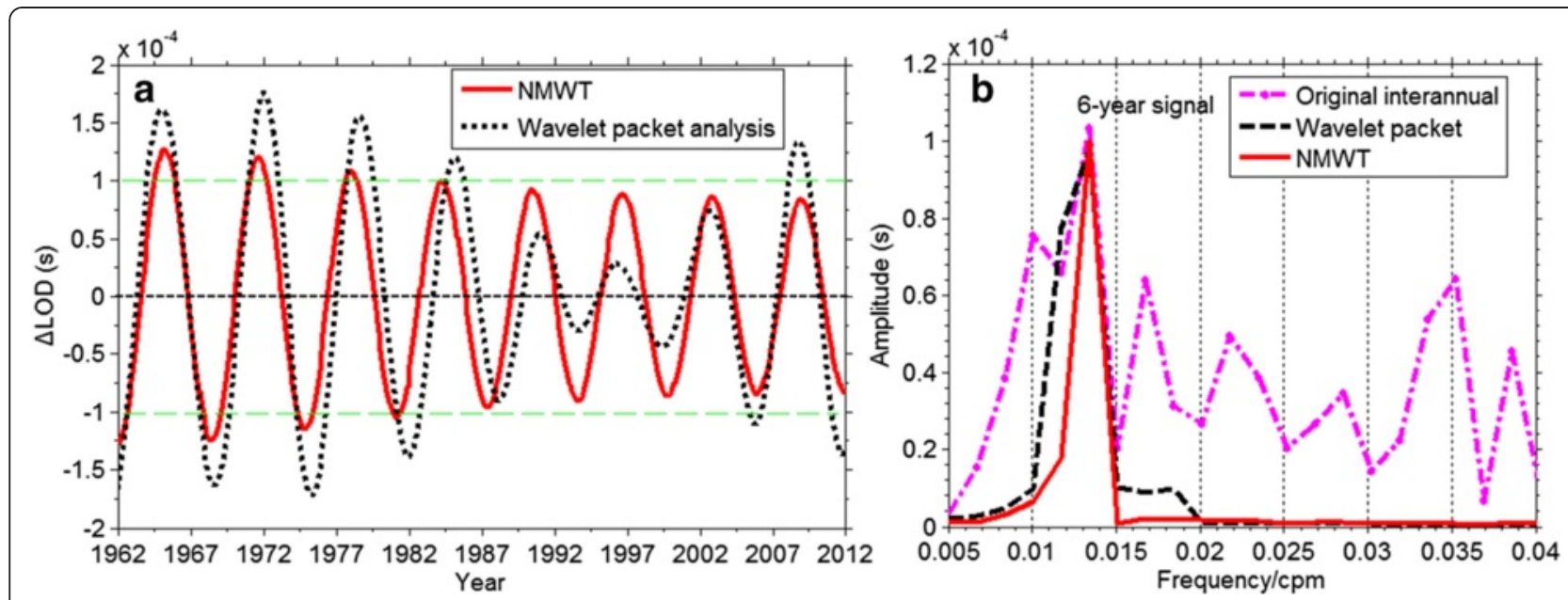

Fig. 7 Comparison of the results from two filtering methods, a time domain and $\mathbf{b}$ frequency domain. a The green dashed lines show the $\pm 0.1 \mathrm{~ms}$; the wavelet packet analysis of the LOD signals on 5 8-year scales showing an abruptly obvious decreasing phenomenon in amplitude during the 1990s, which also can be seen from previous studies; while the result from NMWT shows gradually weakening during the past over 50 years; $\mathbf{b}$ the result from NMWT has a much narrower frequency domain than the result from wavelet packet analysis, which means the result from NMWT should be a very good representative of the pure 6-year signal

Making a further comparison of the 6-year signal before and after the AAM effects were removed, we can see clearly the contribution from AAM effect to the 6year signal (Figs. 9a, b). An obvious conclusion that can be made is that AAM always cancels out the strength of the 6-year signal, which also shows that the contribution from AAM to the 6-year signal is about $0.026 \mathrm{~ms}$, which occupies the mean amplitude of $0.124 \mathrm{~ms}$ about $21 \%$. Figure $9 \mathrm{c}$ suggests that the long-term trend change of the 6-year signal is very consistent with that of the AAM removed, which means the above long-term decreasing phenomenon is unrelated to the AAM effect. Consequently, there should be other uncertain components causing the decreasing phenomenon.

In order to display the results of this paper clearly, we have plotted Fig. 10 with the result from Fig. 6 and the background trend series that has been removed by wavelet filtering.

\section{Discussion}

In this study, we find the 6-year signal in LOD showing the long-term decreasing trend during the past 50 years, which is unrelated to AAM effects. We have a careful review about the result of this paper. In order to test the reliability of the result from NMWT and further verify whether the instantaneous amplitude of the 6-year signal is constant or varying with time, a simple and effective method is used, which is windowed Fourier transform.

We need to compare the relative amplitude values of applying windowed Fourier transform to LOD series (AAM effect has been removed) in different periods. The detailed data preprocessing method is as follows:
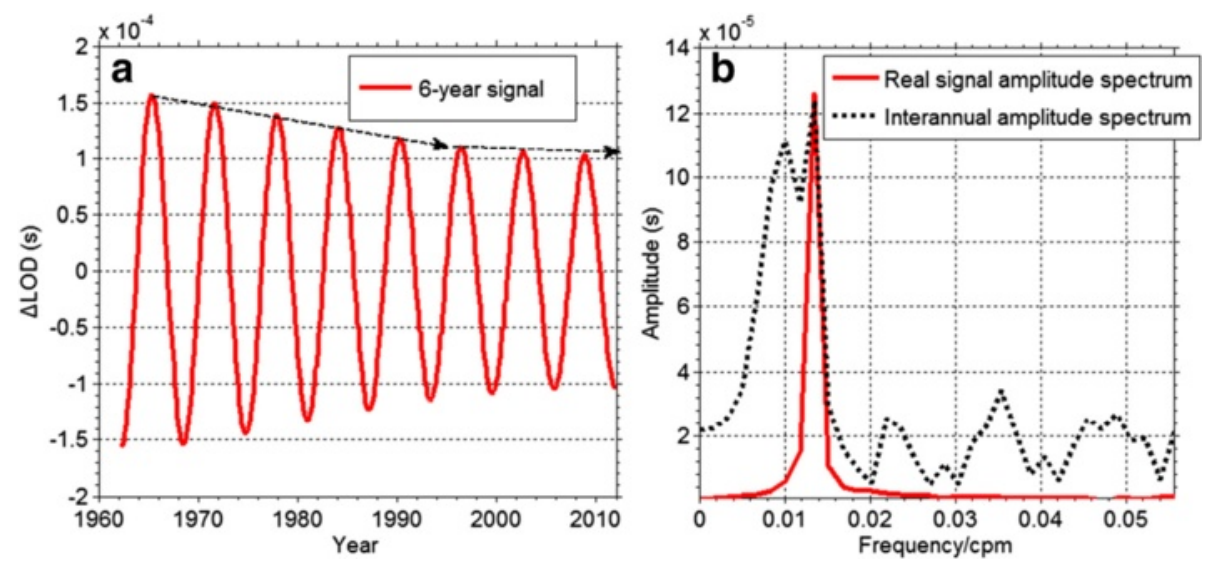

Fig. 8 The 6-year signal of LOD in which AAM effect has been removed, in the time domain (a) and its Fourier spectrum, in the frequency domain (b). From Fig. 8, we can see the long-term amplitude decreasing trend 


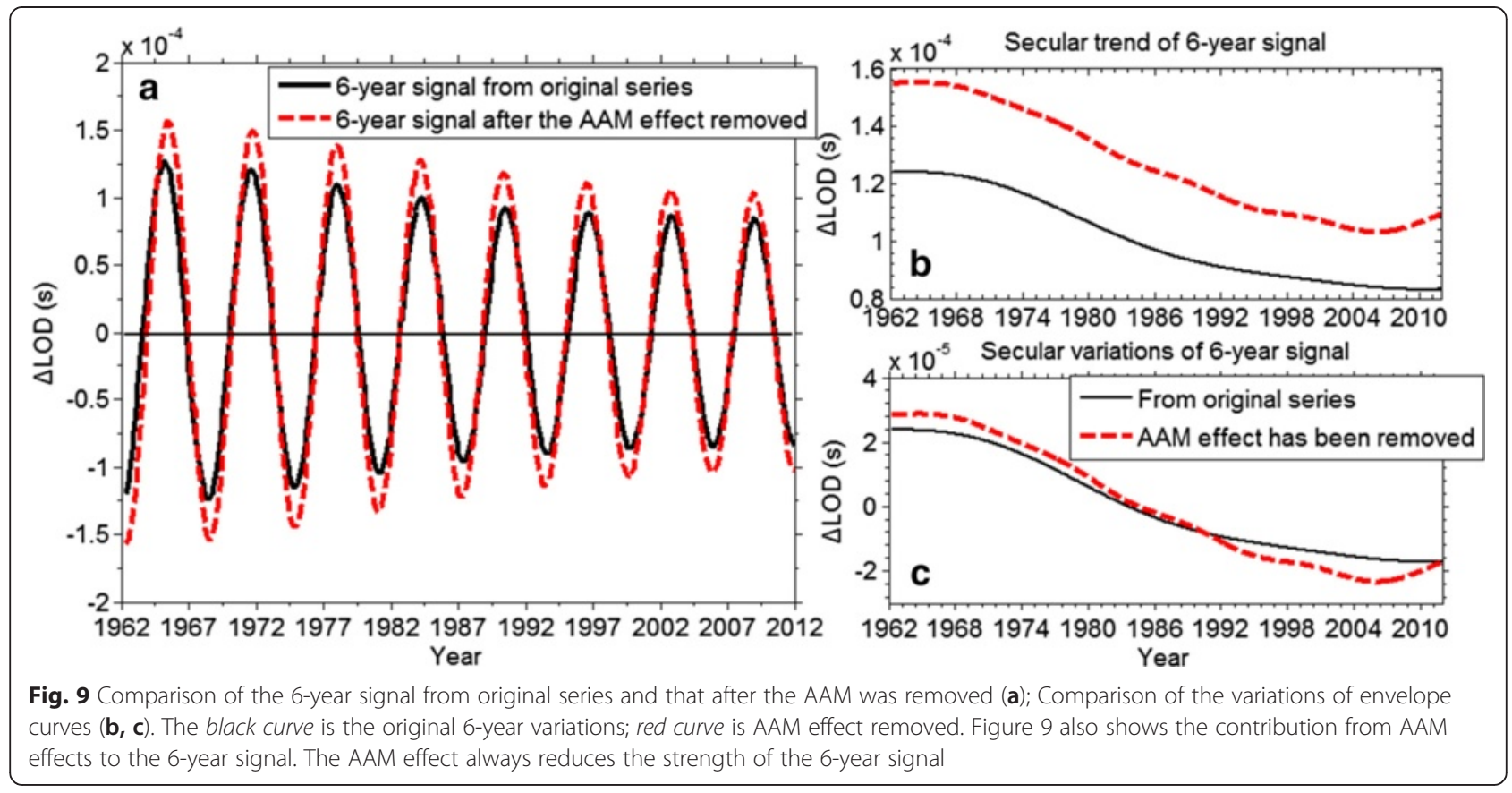

firstly, applying 1-year running average to eliminate the seasonal variations in the LOD series (1962 2012); secondly, fitting the decadal term using 10-order polynomial and remove it from LOD series. We divide the time span (i.e., 1962 2012) into six periods, which are respective $t_{1}$ (1962 1980), $t_{2} \quad(1968 \sim 1986), \quad t_{3} \quad(1974 \sim 1992), \quad t_{4}$ (1980 1998), $t_{5}$ (1986 2004), $t_{6}$ (1992 2010); and then, we compute the normalized Fourier amplitude spectrum one by one in $t_{i}(i=1,2, \ldots 6)$ period and obtain all the spectrum peak values locating at about $0.0138 \mathrm{cpm}$ in each frequency domains (see Fig. 11). Figure 11a shows the comparison of relative amplitude values in $t_{1}$ and $t_{6}$ periods. From Fig. 11a, we also can see that the relative amplitude of the 6-year signal in $t_{1}$ period is about 0.0275 , which is larger than that (about 0.0135 ) in $t_{6}$. The ratio of the former to the latter is about 2 , while the result from NMWT gives the ratio about 1.5 (see Fig. 8a). Because we cannot guarantee that 3 cycles of the 6-year signal can exactly and completely locate each chosen period (i.e., $t_{\mathrm{i}}$ ), some errors in the above method are possible. However, the 6-year signal strength decreasing from $t_{1}$ to $t_{6}$ period is beyond doubt. Figure $11 \mathrm{~b}$ further shows all the relative amplitude values of the 6-year signal in the above six periods and, overall, the spectrum peaks is showing the declining trend, which is consistent with the result of NMWT.

On the other hand, in theory, if the 6-year signal is a stable harmonic signal with an invariant amplitude, it will be recovered precisely by NMWT method with a constant amplitude, which is determined by the properties of NMWT (Liu et al. 2007). Moreover, the longterm reduction is so large that it has occupied the mean amplitude (0.124 ms) about $40 \%$, which means the above phenomenon cannot be simply attributed to the errors produced in data processing or the method this paper uses. However, the result based on the wavelet packet gives us some enlightenment to resolve the debates on the variation feature of the 6-year signal in LOD from previous studies. We propose a viewpoint that the 5 8-year signal from wavelet packet shows a decreasing phenomenon in the 1990s (Fig. 7a), which may be the result of the 6 -year signals beating against

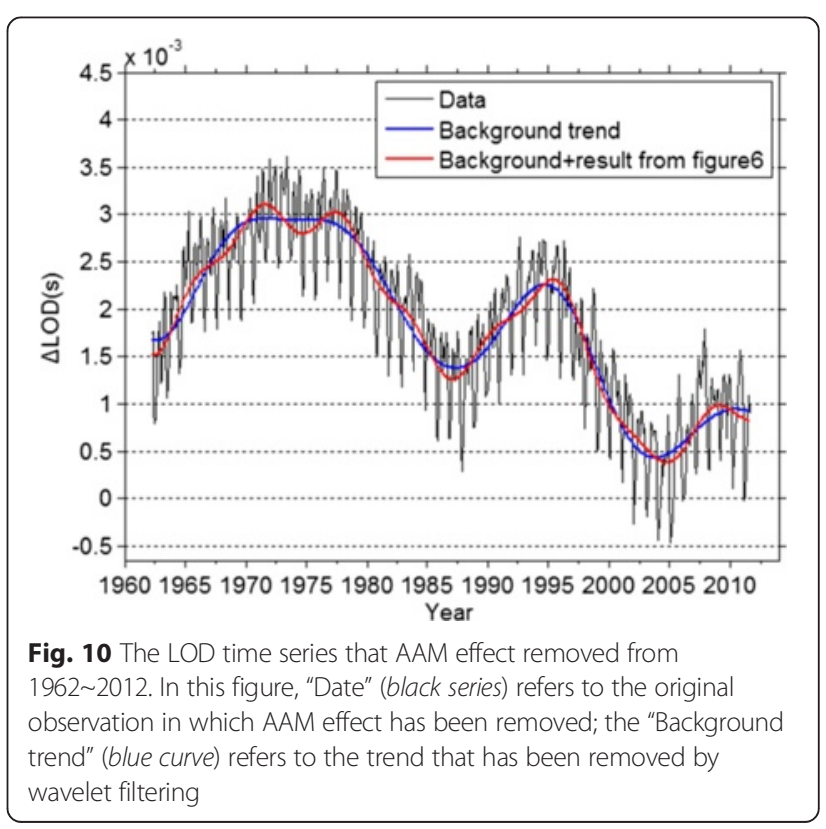



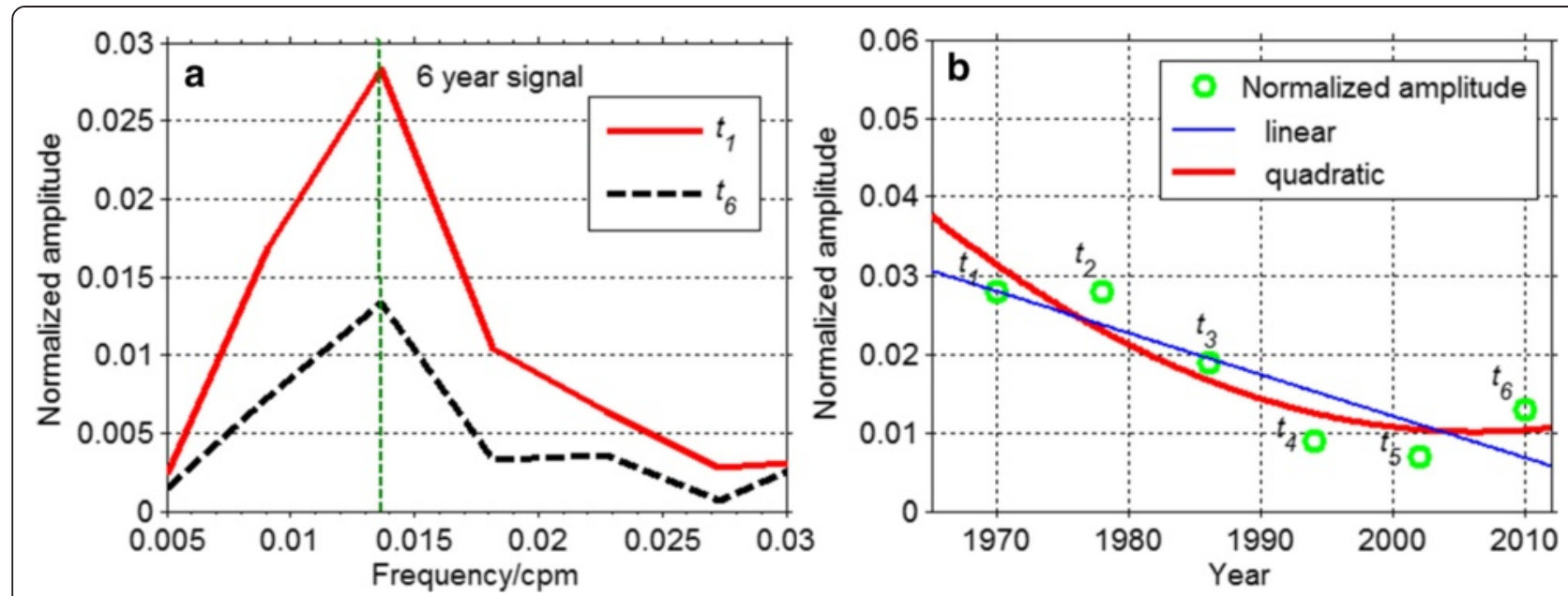

Fig. 11 Comparison of the relative amplitudes of the 6 -year signals in $t_{1}$ and $t_{6}$ periods (a); the total variation trend of the amplitudes in six periods in time domain and the green cycles refer to the relative amplitudes of the 6-year signal in various periods (b); the time window this paper uses is hamming window

the other frequency signals in the 5 8-year bands (see Figs. $8 \mathrm{~b}$ and $7 \mathrm{~b}$ ).

Then, what is the geophysical mechanism of causing the long-term decreasing of the 6-year signal? Many papers (Abarco del Rio et al. 2000, 2003; Chen 2005; Chao and Yan 2010) showed that $\triangle \mathrm{LOD}$ (i.e., the variation of LOD) consists of two terms: motion term and mass term; where motion term contains zonal wind and ocean current excitation; as to the zonal wind excitation, we have removed it from LOD in the AAM. Previous studies (Marcus and Chao 1998) demonstrated that ocean has a low contribution to seasonal variations in LOD (less than $5 \%$ ); its contributions on interannual or longer scales can also be negligible (Abarco del Rio et al.
2000). Thus, we exclude the ocean excitation. Our supposition is that it is mainly related to the mass term of $\triangle \mathrm{LOD}$. The mass term is determined by the mass redistribution and total mass change within the Earth system. The mass term can be expressed as (Chao and William 1988; Bourda 2008):

$$
\Delta \mathrm{LOD}(t)=\frac{2}{3} \frac{M R_{e}^{2}}{C_{m}}\left[\Delta J_{2}(t)+\left(1+k_{2}^{\prime}\right) \frac{\Delta M}{M}\right] \mathrm{LOD}_{\text {mean }}
$$

where $M$ is the Earth's mass of about $5.98 \times 10^{24} \mathrm{~kg}$; $C_{m}$ is the principal moment of inertia of the Earth's mantle of about $7.1 \times 10^{37} \mathrm{kgm}^{2} ; R_{e}$ is the Earth's mean radius of

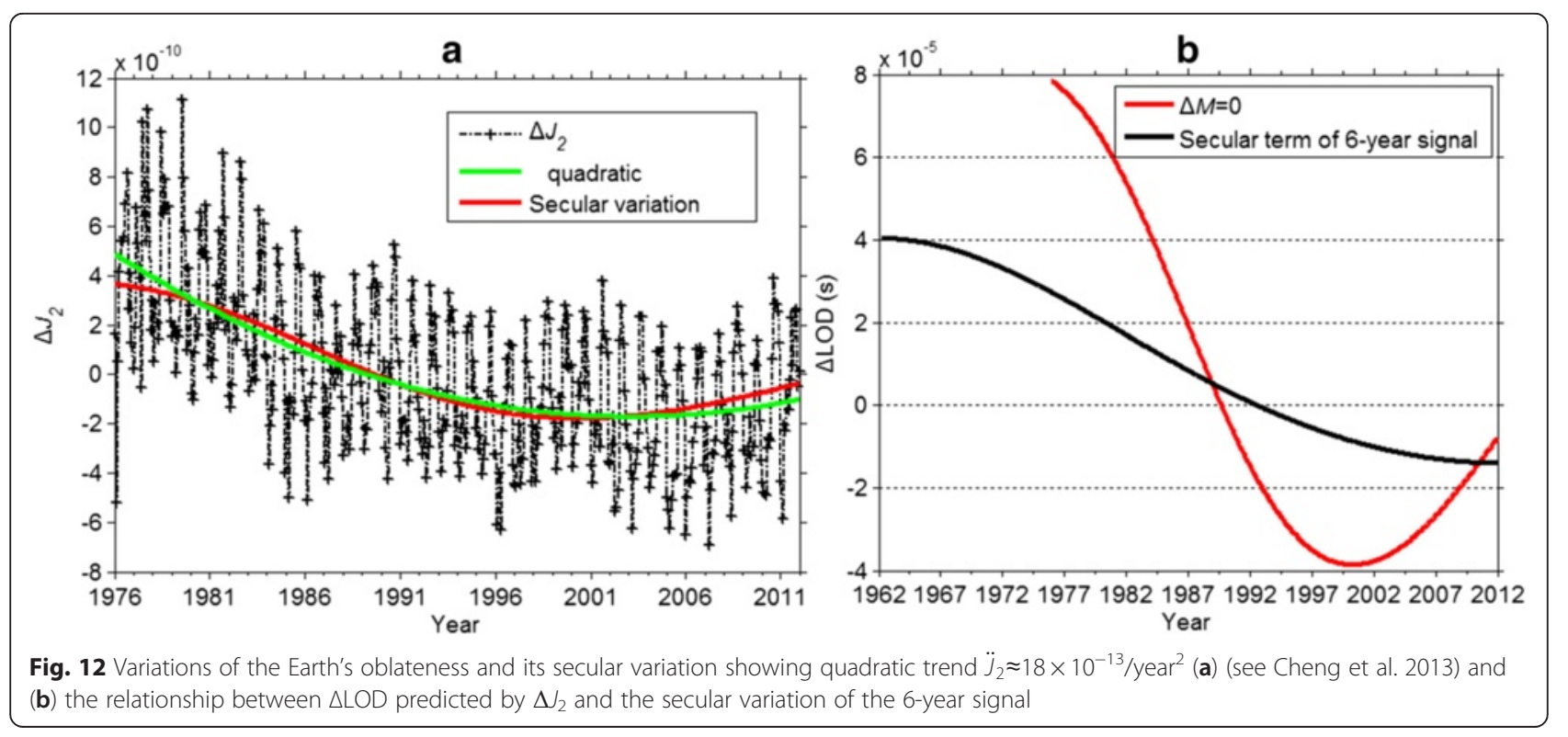


about $6,372,797 \mathrm{~m} ; \Delta J_{2}$ expresses the change of Earth's dynamic oblateness, which is related to the Earth's principal moment of inertia (Cheng et al. 2013; Cheng and Tapley 2004; Cox and Chao 2002; Dickey et al. 2002); $\Delta M$ signifies the Earth's total mass change, which is independent of the mass geographical distribution (Chao and William 1988; Yan and Chao 2012); $k_{2}^{\prime}$ is the love number of -0.31 ; and $\mathrm{LOD}_{\text {mean }}=86400 \mathrm{~s}$. The $\Delta J_{2}$ data is from Cheng et al.(2013) based on Satellite Laser Ranging (SLR) at 30-day intervals from 1976-2011. Figure 12a indicates that the secular variation of $\Delta J_{2}$ is showing a quadratic decreasing trend, but not the linear trend (Cheng et al. 2013).

Formula (9) contains the following two possibilities: The first possibility $\Delta M \equiv 0$, namely the total mass is conserved within the Earth system; then, formula (9) shows the mathematical relationship between $\Delta J_{2}$ and $\Delta$ LOD. We can compute the "equivalent" mass-induced $\triangle \mathrm{LOD}$, because the long-term variation is mainly related to the PGR-poster glacial rebound and melting of the glaciers and ice-sheets mass change (Cheng et al. 2013). Consequently, under the condition of mass conservation, $\triangle \mathrm{LOD}$ is also determined by the same above factors. Figure $12 \mathrm{~b}$ shows that the contribution from mass redistribution within the Earth system is consistent with long-term change of the 6-year signal in trend, but the former decreasing rate is about three times larger than that of the latter. Consequently, there should be other components to prevent the long-term decreasing rate of the 6-year signal.

The second possibilities: $\Delta M \neq 0$, namely, considering of the GMB effect (Yan and Chao 2012). Assuming that the secular decreasing trend of the 6-year signal can be fully interpreted by formula (9), thus, we can get $\Delta M$ within the Earth's system, in turn. By calculation, the secular $\Delta M$ change is in a mean rate of about $+2.1 \times$ $10^{14} \mathrm{~kg} /$ year, from 1976 2011, however, the actual secular variation of $\Delta M$, especially its precise value, is still to be further studied due to its some unknown factors, while the results from Yan and Chao (2012) indicated that the annual amplitude of $\Delta M$ caused by Earth's surface geophysical fluids including atmospheric, oceanic, and land hydrology (i.e., GMB effect) reaches about $41.7 \times 10^{14} \mathrm{~kg}$. Basing from above, the global total mass change $\Delta M$ reaches about $+7.63 \times 10^{15} \mathrm{~kg}$, during 1976 2012. Given that a mean sea level of $1 \mathrm{~cm}$ corresponds to a $\Delta M$ of $3.6 \times 10^{15} \mathrm{~kg}$ (Chao and William 1988), the sea level has risen by $2.1 \mathrm{~cm}$ during the past 36 years. It is essential to estimate accurately the GMB long-term effect for interpreting the secular decreasing phenomena of the 6-year signal.

Then, will the secular decreasing trend of the 6-year signal come from the Earth's core? This is required to be further explored.

\section{Conclusions}

This paper extracts the 6-year temporal signal without AAM effects using NMWT method, quantitatively. The 6-year signal from NMWT shows the following features: 1) the signal with accurate phase, that is, the phase shifting does not happen; 2) the signal with a very narrow frequency range and a high energy concentration degree and a mean amplitude of about $0.124 \mathrm{~ms} ; 3$ ) the secular trend showing a continuous decreasing trend during the past 50 years instead of the abrupt change in some periods. Two possibilities in explaining the long-term decreasing of the 6-year signal are given. We hope this paper can provide a valuable reference for investigating the 6-year signal quantitatively and resolving its geophysical mechanism ultimately.

\section{Competing interests}

The authors declare that they have no competing interests.

\section{Authors' contributions}

PD and GL proposed the topic and the analysis method and also performed analysis; LL, XHu, XHao, and YH collaborated with PD and GL in discussing the analysis method and $L L$ further checked the correctness of the analysis method that this manuscript uses. ZZ and BW carried out the data selection and quality control. All the authors read and approved the final version of the manuscript.

\section{Acknowledgements}

The authors acknowledge the Institute of Geodesy and Geophysics, CAS, for providing computational resources. This work is supported by National Natural Science Foundation of China (grant 41321063, 41374029) and the State Key Laboratory of Geodesy and Earth's Dynamics Foundation (grant SKLGED 2013-4-1-Z).

\section{Author details}

${ }^{1}$ Institute of Geodesy and Geophysics, Chinese Academy of Sciences, State Key Laboratory of Geodesy and Earth's Dynamics, Wuhan 430077, China. 'University of Chinese Academy of Sciences, Beijing 100049, China.

${ }^{3}$ Department of Physics, School of Science, Wuhan University of Technology, Wuhan 430070, China.

Received: 13 June 2015 Accepted: 14 September 2015 Published online: 05 October 2015

\section{References}

Abarco del Rio R, Gambis D, Salstein DA (2000) Interannual signals in length of day and atmospheric angular momentum. Ann Geophys 18:347-364

Abarco del Rio R, Gambis D, Salstein D, Nelson P, Dai A (2003) Solar activity and earth rotation variability. J Geodyn 36:423-443

Bourda G (2008) Length-of-day and space-geodetic determination of the Earth's variable gravity field. J Geod 82:295-305

Chao BF (1989) Length-of-day variations caused by El Nino-southern oscillation and quasi-biennial oscillation. Science 243:923-925

Chao BF, William PO (1988) Effect of uniform sea-level change on the Earth's rotation and gravitational field. Geophys J 93:191-193

Chao BF, Yan HM (2010) Relation between length-of-day variation and angular momentum of geophysical fluids. J Geophys Res 115:B10417. doi:10.1029/ 2009JB007024

Chao BF, Chung WY, Zong R, Shih, Hsieh YK (2014) Earth's rotation variations: a wavelet analysis. Terra Nova 26:260-264

Chen JL (2005) Global mass balance and length-of-day variation. J Geophys Res 110:B08404. doi:10.1029/2004JB003474

Cheng MK, Tapley BD (2004) Variations in the Earth's oblateness during the past 28 years. J Geophys Res 109:B09402. doi:10.1029/2004JB003028

Cheng MK, Tapley BD, Ries JC (2013) Deceleration in the Earth's oblateness. J Geophys Res 118:740-747. doi:10.1002/jgrb.50058 
Cox CM, Chao BF (2002) Detection of a large-scale mass redistribution in the terrestrial system since 1998. Science 297:831-833

Daubechies I (1988) Orthonormal bases of compactly supported wavelet. Commun Pure Appl Math 41:909-996

Davies CJ, Stegman DR, Dumberry M (2014) The strength of gravitational core-mantle coupling. Geophys Res Lett 41, doi:10.1002/2004GL059836

Dickey JO, Marcus SL, Viron O, Fukumori I (2002) Recent Earth oblateness variations: unraveling climate and postglacial rebound effects. Science 298:1975-1977

Gorshkov VL (2010) Study of the interannual variations of the Earth's rotation. Sol Syst Res 44:487-497

Holme R, de Viron O (2005) Geomagnetic jerks and a high-resolution length-of-day profile for core studies. Geophys J Int 160:435-439

Holme R, de Viron O (2013) Characterization and implications of intradecadal variations in length of day. Nature 499:202-205

Hu XG, Liu LT, Hinderer J, Sun HP (2005) Wavelet filter analysis of local atmospheric pressure effects on gravity variations. J Geod 79:447-459

Hu XG, Liu LT, Hinderer J, Sun HP (2006) Wavelet filter analysis of atmospheric pressure effects in the long-period seismic mode band. Phys Earth Planet Inter 154:70-84

Liu LT, Hsu HT, Grafarend EW (2007) Normal Morlet wavelet transform and its application to the Earth's polar motion. J Geophys Res 112:B08401. doi:10.1029/2006JB004895

Mandea M, Holme R, Pais A, Pinheiro K, Jackson A, Verbanac G (2010) Geomagnetic jerks: rapid core field variations and core dynamics. Space Sci Rev 155:147-175

Marcus SL, Chao Y (1998) Detection and modeling of nontidal oceanic effects on Earth's rotation rate. Science 281:1656-1659

Mound JE, Buffett BA (2003) Interannual oscillations in length of day: implications for the structure of the mantle and core. J Geophys Res 108(B7):2334. doi:10.1029/2002JB002054

Mound JE, Buffett BA (2006) Detection of a gravitational oscillation in length-of-day Earth Planet Sci Lett 243:383-389. doi:10.1016/j. epsl.2006.01.043

Silva L, Jackson L, Mound J (2012) Assessing the importance and expression of the 6 year geomagnetic oscillation. J Geophys Res 117:B10101. doi:10.1029/ 2012JB009405

Wang QL, Chen YT, Cui DX, Wang WP, Liang WF (2000) Decadal correlation between crustal deformation and variation in length of day of the Earth. Earth Planets Space 52:989-992

Yan HM, Chao BF (2012) Effect of global mass conservation among geophysical fluids on the seasonal length of day variation. J Geophys Res 117:B02401. doi:10.1029/2011JB008788

Zheng D, Chao BF, Zhou Y, Yu N (2000) Improvement of the edge effect of the wavelet time-frequency spectrum: application to the length-of-day series. J Geod 74:249-254

\section{Submit your manuscript to a SpringerOpen ${ }^{\odot}$ journal and benefit from:}

- Convenient online submission

- Rigorous peer review

- Immediate publication on acceptance

- Open access: articles freely available online

- High visibility within the field

- Retaining the copyright to your article

Submit your next manuscript at springeropen.com 